\title{
HEPATIC PHOSPHOENOLPYRUVATE CARBOXYKINASE GENE EXPRESSION IS NOT REPRESSED BY DIETARY CARBOHYDRATES IN RAINBOW TROUT (ONCORHYNCHUS MYKISS)
}

\author{
S. PANSERAT*, E. PLAGNES-JUAN, J. BRÈQUE AND S. KAUSHIK \\ Laboratory of Fish Nutrition, INRA-IFREMER, 64310 St-Pée-sur-Nivelle, France \\ *e-mail: panserat@st-pee.inra.fr
}

Accepted 27 October 2000; published on WWW 3 January 2001

\begin{abstract}
Summary
Phosphoenolpyruvate carboxykinase (PEPCK) is a ratelimiting enzyme in hepatic gluconeogenesis and therefore plays a central role in glucose homeostasis. The aim of this study was to analyse the nutritional regulation of PEPCK gene expression in rainbow trout (Oncorhynchus mykiss), which are known to use dietary carbohydrates poorly. A full-length hepatic PEPCK cDNA (2637 base pairs with one open reading frame putatively encoding a 635-residue protein) was cloned and found to be highly homologous to mammalian PEPCKs. The presence of a putative peptide signal specific to a mitochondrial-type PEPCK in the deduced amino acid sequence suggests that this PEPCK

gene codes for a mitochondrial form. In gluconeogenic tissues such as liver, kidney and intestine, this PEPCK gene was expressed at high levels and, in the liver we found no regulation of PEPCK gene expression by dietary carbohydrates. These results suggest that the first step of the hepatic gluconeogenic pathway in rainbow trout is functional and highly active irrespective of the dietary carbohydrate supply.

Key words: phosphoenolpyruvate carboxykinase, rainbow trout, Oncorhynchus mykiss, glucose, metabolism, nutrition, gluconeogenesis.
\end{abstract}

\section{Introduction}

Phosphoenolpyruvate carboxykinase (PEPCK; E.C. 4.1.1.32) catalyses the conversion of oxaloacetate to phosphoenolpyruvate and is an important rate-limiting enzymes involved in hepatic gluconeogenesis (Matte et al., 1997). PEPCK is not allosterically or post-transcriptionally modified. Transcriptional regulation of PEPCK gene expression is the major control of PEPCK enzyme activity. The activity of PEPCK (the cytosolic form) is modulated by altering the abundance of the protein, i.e. it is regulated at the transcriptional level by several hormones, whereas the mitochondrial form of hepatic PEPCK is constitutively expressed (Hanson and Reshef, 1997). Cytosolic PEPCK gene transcription is positively regulated by glucagon (through cyclic AMP) and glucocorticoids, whereas it is inhibited by insulin and glucose (Hanson and Reshelf, 1997; Scott et al., 1998; Cournarie et al., 1999). The importance of PEPCK in glucose homeostasis in vivo is also demonstrated by the following: (i) PEPCK gene expression in the liver is inappropriately increased in several diabetic animals (Hanson and Reshelf, 1997), characterised by high hepatic glucose production, and (ii) transgenic animals overexpressing the hepatic PEPCK gene develop insulin resistance and a diabetic phenotype (Valera et al., 1994).

Poor carbohydrate utilisation by rainbow trout (Oncorhynchus mykiss) is considered to be due to the inability of muscle tissue to utilise glucose as an energy source because of the low number of insulin receptors (Parrizas et al., 1994), the low glucose phosphorylation capacity (Cowey and Walton, 1989), the low number of glucose transporters (Wright et al., 1998) or highly active hepatic glucose production from gluconeogenesis. Because PEPCK is the key enzyme controlling hepatic glucose production, the aim of this study was to achieve molecular cloning of the PEPCK cDNA expressed in rainbow trout liver and to analyse the regulation of its expression by dietary carbohydrates.

\section{Materials and methods Fish and diets}

Triplicate groups of juvenile immature rainbow trout Oncorhynchus mykiss (Walbaum) were reared in our experimental fish farm at $18{ }^{\circ} \mathrm{C}$ for 10 weeks during spring under a natural photoperiod. They were fed twice a day to near satiation; one of the experimental diets contained $20 \%$ digestible carbohydrate (supplied as dehulled extruded peas or extruded wheat) and the other was without carbohydrate (Panserat et al., 2000a). Growth rates of fish fed with or without carbohydrates were similar for the two groups (Panserat et al., 2000a): daily growth coefficients $\{[($ final body mass $)^{1 / 3}$ minus (initial body mass) $\left.\left.{ }^{1 / 3}\right] / n\right\} \times 100$, where $n$ is the 
Table 1. Primers used for PEPCK cDNA cloning by RT-PCR (degenerate primers) and RACE-PCR and for PEPCK gene expression analysis by $R T-P C R$

Prime sequences

$\begin{array}{cl}\text { Degenerate primers } & \begin{array}{l}\text { Forward: 5'-TAYRAYAAYTGCTGGYTGGC-3' } \\ \text { Reverse: 5'-CCRAARTTGTAGCCAAARAA-3' }\end{array} \\ \begin{array}{c}\text { Trout-specific primers } \\ \text { 5' RACE-PCR }\end{array} & \begin{array}{l}\text { Forward: kit primer* 1 } \\ \text { Reverse: 5'-TTTCCAGGATTTGCCGTGCC-3' }\end{array} \\ \text { 3' RACE-PCR } & \begin{array}{l}\text { Forward: 5'-GTGGGAAAACTAACCTGGCC-3' } \\ \text { Reverse: kit primer* }\end{array} \\ \text { RT-PCR analysis } & \text { Forward: 5'-GTGGGAAAACTAACCTGGCC-3' } \\ & \text { Reverse: 5'-TCGTCACTCTCCCACTGGGG-3' }\end{array}$

PCR 2

Forward: kit anchored primer*

Reverse: 5'-ACAGTCTTACTCTCCACCCG-3'

Forward: 5'-GTGGGAAAACTAACCTGGCC-3'

Reverse: kit anchored primer*

$\mathrm{Y}, \mathrm{C} / \mathrm{T} ; \mathrm{R}, \mathrm{A} / \mathrm{G}$

*Gene Racer kit (Invitrogen, USA).

number of days on the diet, were $3.31 \pm 0.09$ and $3.36 \pm 0.06$ respectively (means \pm S.D., $N=9$ fish per group). At the end of 10 weeks, after a $24 \mathrm{~h}$ fast, fish (weighing approximately $150 \mathrm{~g}$ ) were fed once, and nine fish from each group were then killed 6 and $24 \mathrm{~h}$ after the meal. Whole liver, kidney, heart, intestine and brain and a small piece of dorsal muscle tissue were removed, clamp-frozen in liquid nitrogen and stored at $-80^{\circ} \mathrm{C}$.

\section{Isolation of RNA}

Total RNA was extracted from rainbow trout tissues as described previously (Chomczinski and Sacchi, 1987). Poly $(\mathrm{A})^{+}$mRNAs were purified from hepatic total RNAs using a poly(dT) column according to the manufacturer's protocol (Promega, USA).

\section{PEPCK cDNA cloning}

\section{Cloning of partial PEPCK CDNA using degenerate primers}

PEPCK sequences from human (GenBank accession number L12760), Drosophila melanogaster (GenBank accession number Y00402) and chicken (GenBank accession numbers M14229 and J05419 for the cytosolic and mitochondrial forms, respectively) were compared using the Clustal-W multiple alignment algorithm (Higgins and Sharp, 1989). Degenerate primers were chosen on the basis of the most conserved coding regions of PEPCK at positions 460-479 and 1698-1717 of the cytosolic PEPCK gene from chicken. The sequences of the degenerated primers are shown in Table 1. Hepatic cDNA was obtained by annealing $2 \mu \mathrm{g}$ of total RNA with $1 \mu \mathrm{g}$ of random primers and incubating with AMV reverse transcriptase (Boehringer, Roche Molecular Biochemicals, Germany) for $1 \mathrm{~h}$ at $42^{\circ} \mathrm{C}$. cDNA $(1 \mu \mathrm{l})$ was amplified by polymerase chain reaction (PCR), using $100 \mathrm{pmol}$ of the degenerate primers, in a reaction mixture containing $2 \mathrm{mmoll}^{-1} \mathrm{MgCl}_{2}, 50 \mathrm{mmoll}^{-1}$ $\mathrm{KCl}, 20 \mathrm{mmol}^{-1}$ Tris- $\mathrm{HCl}, 0.25 \mathrm{mmoll}^{-1} \mathrm{dNTP}$ and 2.5 units of Taq polymerase (Boehringer, Roche Molecular Biochemicals, Germany). Thirty-five cycles of denaturation for $1 \mathrm{~min}$ at $94^{\circ} \mathrm{C}$, annealing at $52^{\circ} \mathrm{C}$ for $40 \mathrm{~s}$ and extension at $72{ }^{\circ} \mathrm{C}$ for $1 \mathrm{~min}$ were performed. PCR products were subjected to electrophoresis in $1 \%$ agarose gels, and fragments of the expected size range (1300 base pairs, bp) were purified (Micropure System, Amicon, USA). The purified DNA fragments were inserted into the pCRII plasmid and used for transformation of One Shot competent cells (Invitrogen, Carlsbad, CA, USA). Inserts were detected by EcoRI digestion of the extracted plasmid DNA. Clones with inserts were sequenced (Cybergène, Evry, France).

Reverse transcription (RT), rapid amplification of the cDNA extremities-polymerase chain reaction (RACE-PCR) and molecular cloning of PCR fragments

The $5^{\prime}$ and $3^{\prime}$ cDNA extremities were determined by the RACE-PCR method as detailed in the manufacturer's protocol (Gene Racer kit, Invitrogen, USA). Trout-specific PEPCK primers were designed from the partial sequence (Table 1). Using the reverse transcription system, cDNA was synthesised by incubating $1 \mu \mathrm{g}$ of hepatic poly(A) ${ }^{+}$mRNA with AMV reverse transcriptase for $1 \mathrm{~h}$ at $42^{\circ} \mathrm{C}$ using either the oligo(dT) primer ( $\left.3^{\prime} \mathrm{RACE}\right)$ or a trout-specific PEPCK primer $5^{\prime}$ TCGTCACTCTCCCACTGGGG- $3^{\prime}$ (5' RACE). The two RACE-PCR reactions were carried out using trout-specific PEPCK primers at annealing temperatures of $60^{\circ} \mathrm{C}$ and $57^{\circ} \mathrm{C}$ for $5^{\prime}$ and $3^{\prime}$ RACE-PCR, respectively (Table 1). PCR products were subjected to electrophoresis in $1 \%$ agarose gels and hybridised with ${ }^{32} \mathrm{P}$-labelled PEPCK probes, and the relevant fragments were purified (Micropure System, Amicon, USA), cloned (Invitrogen, USA) and sequenced (Cybergène, Evry, France) as described above.

\section{Sequence analysis}

Nucleotide sequences (excluding the primer sequences) were compared with DNA sequences from the Genbank database using the basic local alignment search tool (BLAST) algorithm (Altschul et al., 1990). Sequence alignments and percentage conservation of amino acid sequences were assessed with the Clustal-W multiple-alignment algorithm (Higgins and Sharp, 1989) using the trout PEPCK sequence and known PEPCK sequences from databases. The search for a peptide signal in trout PEPCK cDNA sequence was assessed 
GAAAGCAGTTCTTCCTCATAGACCAATCAGATATGAGTTGTAGTTGGTOCTAAAGGGCACACGACOCTCTT 71 ATTCTСТСТСТСТСТАTATATATTTATTTTTTTAGACTTTTATATTTCATTACCCCATTTTAGTATTTTTAC 143 $\begin{array}{cccccccccccccccccc}\text { M } & \text { S } & \text { C } & \text { L } & \text { L } & \text { L } & \text { G } & \text { L } & \text { I } & \text { R } & \text { R } & \text { R } & \text { G } & \text { G } & \text { V } & \text { G } & \text { T } & 17 \\ \text { AAG } & \text { ATG TCG TOC } & \text { CTT } & \text { TTG } & \text { CTT } & \text { GGA } & \text { CTT } & \text { ATC } & \text { AGA } & \text { AGA } & \text { COG } & \text { OGT } & \text { OGA } & \text { GTG } & \text { GOG } & \text { ACA } & 197\end{array}$ $\begin{array}{lllllllllllllllllll}\text { S } & \text { V } & \text { G } & \text { V } & \text { R } & \text { S } & \text { L } & \text { A } & \text { S } & \text { I } & \text { P } & \text { S } & \text { L } & \text { P } & \text { P } & \text { A } & \text { V } & \text { A } & 35\end{array}$ $\begin{array}{llllllllllllllllllll}\text { D } & \text { F } & \text { V } & \text { K } & \text { R } & \text { A } & \text { V } & \text { D } & \text { E } & \text { C } & \text { K } & \text { P } & \text { A } & \text { N } & \text { V } & \text { H } & \text { V } & \text { V } & & 53\end{array}$ GAC TTT GTG AAG AOG GCC GTG GAT GAG TOC AAG OCT OCC AAT GTG CAT GTG GTG 305 $\begin{array}{ccccccccccccccccccc}\text { T } & \text { G } & \text { S } & \text { A } & \text { E } & \text { E } & \text { S } & \text { A } & \text { H } & \text { I } & \text { L } & \text { A } & \text { G } & \text { L } & \text { E } & \text { K } & \text { D } & \text { G } & 71 \\ \text { ACG GOG AOC } & \text { OCG } & \text { GAG } & \text { GAG } & \text { TOC } & \text { OCT } & \text { CAC } & \text { ATC } & \text { CTA } & \text { OCT } & \text { OOC } & \text { CTG } & \text { GAG AAA } & \text { GAC } & \text { GOC } & 359\end{array}$ $\begin{array}{ccccccccccccccccccr}\text { M } & \text { V } & \text { K } & \text { R } & \text { L } & \text { P } & \text { K } & \text { Y } & \text { E } & \text { N } & \text { C } & \text { W } & \text { L } & \text { A } & \text { R } & \text { T } & \text { D } & \text { P } & 89 \\ \text { ATG GTG AAG AOG } & \text { CTA CCC } & \text { AAG TAT } & \text { GAG } & \text { AAC } & \text { TOC } & \text { TOG } & \text { CTG } & \text { OCA } & \text { CGT } & \text { ACA } & \text { GAC } & \text { COC } & 413\end{array}$ $\begin{array}{llllllllllllllllllll}\text { K } & \text { D } & \text { V } & \text { A } & \text { R } & \text { V } & \text { E } & \text { S } & \text { K } & \text { T } & \text { V } & \text { I } & \text { V } & \text { T } & \text { K } & \text { N } & \text { Q } & \text { R } & & 107\end{array}$ AAG GAC GTG OCT COG GIG GAG AGT AAG ACT GTG ATC GTC ACC AAG AAC CAG AGG 467 $\begin{array}{ccccccccccccccccccc}\text { D } & \text { T } & \text { I } & \text { P } & \text { I } & \text { P } & \text { D } & \text { G } & \text { G } & \text { A } & \text { K } & \text { S } & \text { Q } & \text { L } & \text { G } & \text { S } & \text { W } & \text { M } & 125 \\ \text { GAC ACC } & \text { ATC } & \text { OCT } & \text { ATC } & \text { COC } & \text { GAT } & \text { GGG } & \text { GOG } & \text { OCT } & \text { AAG } & \text { AOC } & \text { CAG } & \text { CTG } & \text { GOC } & \text { AOC } & \text { TOG } & \text { ATG } & 52\end{array}$ $\begin{array}{ccccccccccccccccccc}\text { S } & \text { E } & \text { G } & \text { D } & \text { F } & \text { Q } & \text { K } & \text { A } & \text { R } & \text { Q } & \text { D } & \text { R } & \text { F } & \text { P } & \text { G } & \text { C } & \text { M } & \text { S } & 143 \\ \text { AGT } & \text { GAG OGT } & \text { GAC } & \text { TTC CAG } & \text { AAG } & \text { OCC } & \text { AGA } & \text { CAG } & \text { GAC } & \text { COC } & \text { TTC } & \text { CCA } & \text { OOC } & \text { TOC } & \text { ATG } & \text { TCA } & 575\end{array}$ $\begin{array}{ccccccccccccccccccc}\text { G } & \text { R } & \text { T } & \text { M } & \text { Y } & \text { V } & \text { I } & \text { P } & \text { F } & \text { S } & \text { M } & \text { G } & \text { P } & \text { V } & \text { G } & \text { S } & \text { P } & \text { L } & 161 \\ \text { OGT CGA ACC } & \text { ATG TAT } & \text { GTG } & \text { ATC } & \text { CCC } & \text { TTC } & \text { AOC } & \text { ATG } & \text { GOC } & \text { CCG } & \text { GTG } & \text { OOC } & \text { TCT } & \text { CCG } & \text { CTG } & 629\end{array}$ $\begin{array}{ccccccccccccccccccc}\text { S } & \text { K } & \text { F } & \text { G } & \text { V } & \text { Q } & \text { V } & \text { T } & \text { D } & \text { S } & \text { P } & \text { Y } & \text { V } & \text { V } & \text { A } & \text { S } & \text { M } & \text { G } & 179 \\ \text { TCT } & \text { AAG TTT } & \text { GOC } & \text { GTG CAG } & \text { GTG ACA } & \text { GAC } & \text { TCA } & \text { COC } & \text { TAC } & \text { GTG } & \text { GTG GCC } & \text { AGT } & \text { ATG GOC } & 683\end{array}$

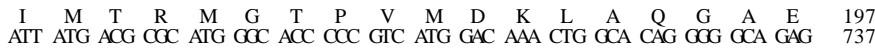
$\begin{array}{ccccccccccccccccccc}\text { F } & \text { V } & \text { R } & \text { C } & \text { Q } & \text { H } & \text { S } & \text { L } & \text { G } & \text { R } & \text { P } & \text { L } & \text { P } & \text { L } & \text { K } & \text { A } & \text { P } & \text { L } & 215 \\ \text { TTT GTA COC TOC CAG } & \text { CAC } & \text { TOC } & \text { CTC } & \text { OGT } & \text { COG } & \text { COC } & \text { CTC } & \text { OCA CTG AAA } & \text { OCT } & \text { COC } & \text { CTG } & 791\end{array}$ $\begin{array}{ccccccccccccccccccc}\text { V } & \text { N } & \text { S } & \text { W } & \text { P } & \text { C } & \text { N } & \text { P } & \text { E } & \text { K } & \text { V } & \text { L } & \text { I } & \text { S } & \text { H } & \text { L } & \text { P } & \text { D } & 233 \\ \text { GTC AAC TCG TOG } & \text { CCG TGT } & \text { AAC } & \text { OCA } & \text { GAG } & \text { AAG } & \text { GTG } & \text { CTG } & \text { ATC } & \text { TOC } & \text { CAC } & \text { CTG OCA GAC } & 845\end{array}$ $\begin{array}{ccccccccccccccccccc}\text { T } & R & \text { Q } & \text { I } & \text { L } & \text { S } & \text { F } & \text { G } & \text { S } & \text { G } & \text { Y } & \text { G } & \text { G } & \text { N } & \text { S } & \text { L } & \text { L } & \text { G } & 251 \\ \text { AOC } & \text { AGG CAG } & \text { ATC CTG TCG TTC } & \text { GOC } & \text { AGT } & \text { GOC } & \text { TAC } & \text { GGA } & \text { OGC } & \text { AAC } & \text { TCC } & \text { CTG CTG } & \text { GOG } & 899\end{array}$ $\begin{array}{lllllllllllllllllll}\text { K } & \text { K } & \text { C } & \text { F } & \text { A } & \text { L } & \text { R } & \text { I } & \text { A } & \text { S } & \text { R } & \text { I } & \text { A } & \text { K } & \text { D } & \text { E } & \text { G } & \text { W } & 269\end{array}$ $\begin{array}{llllllllllllllllllllll}\text { L } & \text { A } & \text { E } & \text { H } & \text { M } & \text { L } & \text { I } & \text { L } & \text { G } & \text { I } & \text { T } & \text { N } & \text { P } & \text { Q } & \text { G } & \text { V } & \text { K } & \text { R } & 287\end{array}$ $\begin{array}{ccccccccccccccccccr}\text { Y } & \text { V } & \text { A } & \text { A } & \text { A } & \text { F } & \text { P } & \text { S } & \text { A } & \text { C } & \text { G } & \text { K } & \text { T } & \text { N } & \text { L } & \text { A } & \text { M } & \text { M } & 305\end{array}$ $\begin{array}{ccccccccccccccccccc}\text { K } & \text { P } & \text { A } & \text { L } & \text { P } & \text { G } & \text { W } & \text { T } & \text { V } & \text { E } & \text { C } & \text { V } & \text { G } & \text { D } & \text { D } & \text { I } & \text { A } & \text { W } & 323 \\ \text { AAG CCA GCG } & \text { CTG } & \text { OCT } & \text { GOC } & \text { TOG } & \text { ACT } & \text { GTG } & \text { GAG } & \text { TGT } & \text { GTA } & \text { OGA } & \text { GAC } & \text { GAC } & \text { ATC } & \text { OCC } & \text { TGG } & 1115\end{array}$ $\begin{array}{rrrrrrrrrrrrrrrrrrr}\text { M } & \text { K } & \text { F } & \text { D } & \text { S } & \text { Q } & \text { G } & \text { K } & \text { L } & \text { R } & \text { A } & \text { I } & \text { N } & \text { P } & \text { E } & \text { N } & \text { G } & \text { F } & 341 \\ \text { ATG AAG TTC } & \text { GAC } & \text { AGT } \\ \text { CAG } & \text { GGT } & \text { AAA } & \text { CTC } & \text { AOG } & \text { OCA } & \text { ATC } & \text { AAC } & \text { CCA } & \text { GAG } & \text { AAC } & \text { OOC } & \text { TTT } & 1169\end{array}$ $\begin{array}{lllllllllllllllllll}\text { F } & \text { G } & \text { V } & \text { A } & \text { P } & \text { G } & \text { T } & \text { S } & \text { L } & \text { K } & \text { T } & \text { N } & \text { P } & \text { H } & \text { A } & \text { M } & \text { A } & \text { T } & 359\end{array}$ $\begin{array}{lllllllllllllllllll}\text { I } & \text { A } & \text { K } & \text { N } & \text { T } & \text { V } & \text { F } & \text { T } & \text { N } & \text { V } & \text { G } & \text { E } & \text { T } & \text { S } & \text { D } & \text { G } & \text { G } & \text { V } & 377\end{array}$

using the SignalIP algorithm (Center for Biological Sequence Analysis, Denmark) (Nielsen et al., 1997).

\section{PEPCK gene expression}

\section{Northern analysis}

Extracted total RNA samples $(20 \mu \mathrm{g})$ were electrophoresed in $1 \%$ agarose gels containing $5 \%$ formaldehyde and capillary-transferred onto nylon membrane (Hybond-N ${ }^{+}$, Amersham, England). After transfer, RNA blots were stained with Methylene Blue to locate $26 \mathrm{~S}$ and $16 \mathrm{~S}$ rRNAs and to determine the amount of loaded RNA. Membranes were hybridised with ${ }^{32} \mathrm{P}$-labelled DNA probes labelled by random priming (Stratagene, USA) recognising partial rainbow trout PEPCK cDNA (1262 bp). After stringent washing, the membranes were exposed to X-ray film and the resulting images were quantitated using Visio-Mic II software (Genomic, France).

\section{$R T-P C R$ analysis}

cDNAs (produced from total RNA extracted in different tissues) were amplified by PCR using specific primers chosen from the rainbow trout PEPCK cDNA sequence (Table 1). The $\begin{array}{ccccccccccccccccccc}\text { W } & \text { W } & \text { E } & \text { G } & \text { L } & \text { D } & \text { P } & \text { P } & \text { A } & \text { A } & \text { G } & \text { V } & \text { S } & \text { L } & \text { T } & \text { D } & \text { W } & \text { H } & 395 \\ \text { TOG TOG GAG GGA CTG GAC OCC } & \text { OCT } & \text { OCC } & \text { OCA } & \text { OGG GTC TOC } & \text { CTG ACC GAC } & \text { TOG } & \text { CAC } & 1331\end{array}$ $\begin{array}{lllllllllllllllllll}\text { G } & \text { K } & \text { S } & \text { W } & \text { K } & \text { A } & \text { G } & \text { D } & \text { S } & \text { G } & \text { P } & \text { C } & \text { A } & \text { H } & \text { P } & \text { N } & \text { S } & \text { R } & 413\end{array}$ $\begin{array}{ccccccccccccccccccc}\text { F } & \text { C } & \text { T } & \text { P } & \text { A } & \text { A } & \text { Q } & \text { C } & \text { P } & \text { I } & \text { I } & \text { D } & \text { P } & \text { P } & \text { W } & \text { E } & \text { S } & \text { D } & 431 \\ \text { TTC TGT } & \text { AOC } & \text { COG } & \text { OCG } & \text { OCC CAG TOC } & \text { COC } & \text { ATC } & \text { ATC GAC } & \text { GCC } & \text { CAG TOG } & \text { GAG AGT } & \text { GAC } & 1439\end{array}$

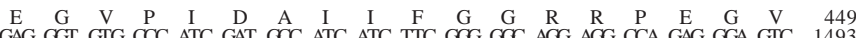

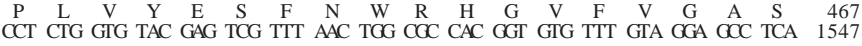
$\begin{array}{lllllllllllllllllll}M & R & \text { S } & \text { E } & \text { A } & \text { T } & \text { A } & \text { A } & \text { A } & \text { E } & \text { Y } & \text { K } & \text { G } & \text { K } & \text { V } & \text { I } & \text { M } & \text { H } & 485\end{array}$ ATG AGG TCT GAG OCC ACA OCA OCT OCT GAG TAC AAA GOC AAG GIT ATC ATG CAC 1601

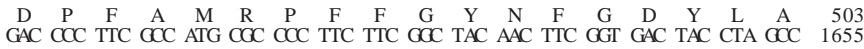

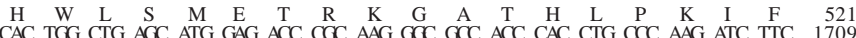
$\begin{array}{ccccccccccccccccccc}\text { H } & \text { V } & \text { N } & \text { W } & \text { F } & \text { R } & \text { K } & \text { D } & \text { P } & \text { T } & \text { S } & \text { G } & \text { S } & \text { F } & \text { L } & \text { W } & \text { P } & \text { G } & 539 \\ \text { CAC GIC } & \text { AAC TOG } & \text { TTC COG } & \text { CAG } & \text { GAC COC } & \text { ACG TOG } & \text { TOC } & \text { TCT } & \text { TTC CTC TOG COC OGT } & 1763\end{array}$

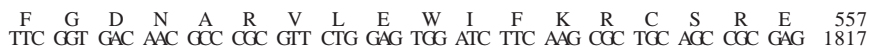
$\begin{array}{lllllllllllllllllll}R & \text { E } & \text { D } & \text { E } & \text { A } & \text { A } & \text { K } & \text { K } & \text { S } & \text { M } & \text { V } & \text { G } & \text { W } & \text { V } & \text { P } & \text { L } & \text { E } & \text { G } & 575\end{array}$ GCC TGG GTG CCA CTG GAG GGA 1871

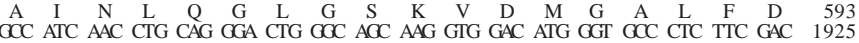
$\begin{array}{ccccccccccccccccccc}\text { L } & \text { P } & \text { K } & \text { A } & \text { F } & \text { W } & \text { E } & \text { K } & \text { E } & \text { T } & \text { Q } & \text { E } & \text { L } & \text { R } & \text { A } & \text { Y } & \text { F } & \text { T } & 611 \\ \text { CTG COC } & \text { AAG OCC TTC TOGG GAG AAG GAG } & \text { ACC CAG GAG CTG } & \text { AOG } & \text { OCG TAC } & \text { TTT } & \text { ACC } & 1979\end{array}$

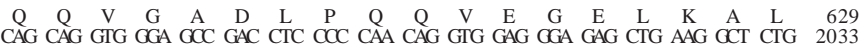
$\begin{array}{llllllll}E & D & R & I & R & N & *\end{array}$

MAT GAG GTG GAG GGA CCA GAT GAT TCA GAG AGA GAA 2035 AGT ATT GAC TAT TCG ACA TTA TAC TAC GST GAT OCT GCT AAA TCA TAT OGT AGT 2141 ACT GAC AAA AAA TTA CTA ACA AOC TMA TTT CAA TTT TGT OGA AGC TOC TTT OCC 2125 TAT ATG TGG GTA GAT GTA AAC CAA AGT TTC TAA ATT GTA AGA AAA TAC AAT GAG 2249 ATG ATG TGA CTG CAT CAC TGA AGA ATG CAG TTC ATG CTA TCA CAC ACT GIC AGT 2303 GOC TAT ATC AGT AGG TTG TAG GTG ATG GAG CAG AAT COG TGT TTA GCA GTA CCT 2357 TAC TGA CAT CTT OCA AAG AAG TAT AAA GGT ATT GGA TGT TTA CAA OCT TTA OCC 2411 CAA ATA ATC TAA TCT AAT CAG GAC AAG CTT AAA TAT CCA TAT TCA TTT AAA CAT 2465 TTT TAA ACA OCA TTT TTT TTG TCA CTT TTG TAT TGT TTC CAA TAA AAA TGA TTA 2519 TGA OCT AAT GTT TAC ACA TGA GAT ATG TAA CAT GTT GGA CCA ATA AAA ATG ATT 2573 AAA AAA AAA A

Fig. 1. Nucleotide sequence of rainbow trout phosphoenolpyruvate carboxykinase (PEPCK) cDNA and deduced amino acid sequences. Italic, underlined letters correspond to the poly $(\mathrm{A})^{+}$cleavage signal. Bold, underlined letters indicate the first amino acid residue and an asterisk the codon stop.

PCR reaction was carried out in a final volume of $25 \mu \mathrm{l}$ containing $1.5 \mathrm{mmoll}^{-1} \mathrm{MgCl}_{2}, 4 \mathrm{pmol}$ of each primer, $2 \mu \mathrm{l}$ of cDNA and 1 unit of Taq polymerase (Boehringer, Roche Molecular Biochemicals, Germany). PCR was performed using 35 cycles of $20 \mathrm{~s}$ for hybridisation (at $59^{\circ} \mathrm{C}$ ), $20 \mathrm{~s}$ for elongation (at $72{ }^{\circ} \mathrm{C}$ ) and $20 \mathrm{~s}$ for denaturation (at $94^{\circ} \mathrm{C}$ ). The PCR products were characterised by hybridisation with the ${ }^{32} \mathrm{P}$-labelled DNA rainbow trout PEPCK probe.

\section{Data analysis}

Results are expressed as means \pm standard deviation (S.D.). Statistically significant differences between the two series of data were assessed using an unpaired two-tailed Student's $t$-test (Systat 9 software, SPSS Inc., USA). For multiple comparisons, data were analysed by one-way analysis of variance (ANOVA; Systat 9 software, SPSS Inc., USA). Differences were considered significant at $P<0.05$.

\section{Results}

RT-PCR was performed on hepatic total RNA, extracted from fish fed without carbohydrates, using degenerate 
PEPCK-C-human PEPCK-C-chicken PEPCK-trout PEPCK-M-chicken

PEPCK-C-human PEPCK-C-chicken PEPCK-trout PEPCK-M-chicken

PEPCK-C-human PEPCK-C-chicken PEPCK-trout PEPCK-M-chicken

PEPCK-C-human PEPCK-C-chicken PEPCK-trout PEPCK-M-chicken

PEPCK-C-human PEPCK-C-chicken PEPCK-trout PEPCK-M-chicken

PEPCK-C-human PEPCK-C-chicken PEPCK-trout PEPCK-M-chicken

PEPCK-C-human PEPCK-C-chicken PEPCK-trout PEPCK-M-chicken

PEPCK-C-human PEPCK-C-chicken PEPCK-trout PEPCK-M-chicken

Fig. 2. Comparison of the rainbow trout phosphoenolpyruvate carboxykinase (PEPCK), human liver cytosolic PEPCK (GenBank accession number L12760) and chicken cytosolic (C) and mitochondrial (M) PEPCKs (GenBank accession numbers M14229 and J05419, respectively) amino acid sequences deduced from PEPCK cDNA sequences. Asterisks mark amino acid residues homologous between all these PEPCKs. The shaded boxes represent the motifs conserved in all these PEPCKs. Predicted mitochondrial targeting sequences (determined by the SignalIP algorithm) are underlined.

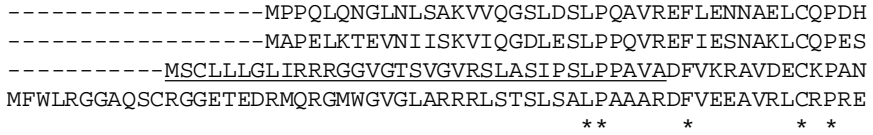

IH ICDGSEEENGRLLGQMEEEGILRRLKKYDNCWLALTDPRDVARIESKTVIVTQEQRDT I H ICD GSEEENKKI LD IMVEOGMI KKLSKYENCWLALTNPRDVARIESKTVI ITOEORDT VHVVTGSAEESAHI LAGLEKDGMVKRLP KYENCWLARTDPKDVARVESKTVIVTKNQRDT VLLCDGSEEEGKELLRGLQDDGVLHP LPKYDNCWLARTDPRDVARVQSKTVLVTPEQSDA

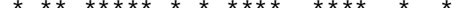

VP IPKTGLS-QLGRWMSEEDFEKAFNARFPGCMKGRTMYVIPFSMGPLGSP LSKIGIELT IP IPKTGSS-OLGRWMSEEDFEKAFNTRFPGCMOGRTMYVIPFSMGP IGSP LAKIGIELT IP IPDGGAKSQLGSWMSEGDFQKARQDRFPGCMSGRTMYVIPFSMGPVGSP LSKF GVQVT VPPPPP SGSPQLGNWMSP NAFQAAVQERFPGCMAGRPLYVIP FSMGPP TSP LAKLGVQVT

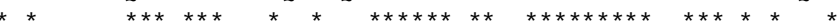

DSPYVVASMRIMTRMGTPVLEALGD-GEFVKCLHSVGCP LP LQKP LVNNWP CNPELTLIA DSPYVVASMRMMTRMGTAALKALGN-GEFVKCLHSVGCP LP LKEP L INNWP CNP ELTL IA DSPYVVASMGIMTRMGTPVMDKLAQGAEFVRCQHS LGRP LP LKAP LVNSWP CNP EKVL IS DSPYVVLSMRIMTRVGPAVLQRLDD--DFVRCLHSVGRP LP LTEP LVSSWP CDRSRVLVA

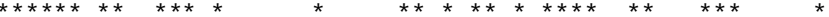
PEPCK-specific domain

HLPDRREI I S FSGYGGNSLLGKK CFALRNASRLAKEEGWLAEHML ILG ITNPEGEKKYL HLPDRREI ISH GSGYGGNS LLGKKCF ALRIASRIAKEEGWLAEHMLILGITNPEGEKKYF HLPDTRQI LSE GSGYGGNS LLGKKCF ALRIASRIAKDEGWLAEHML ILGITNPQGVKRYV HIP SERRIVSE GSGYGGNSLLGKKCFALAIASRMAQQQGWLAEHMLILGVTSPSGEKRYM

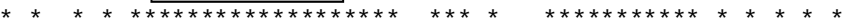

Kinase-1 Kinase-2

AAA FP SACGK\$NLAMMNP S LP GWKVE CVGDD I AWMKFDAQGHLRA INP ENGF FGVAP GTS AAA FP SACGK NLAMMNP SRP GWK I E CVGDD I AWMKFDELGNLRA I NP ENGF FGVAP GTS AAA FP SACGK NLAMMKP ALP GWTVE CVGDD I AWMKFD SQGKLRA INP ENGF FGVAP GTS

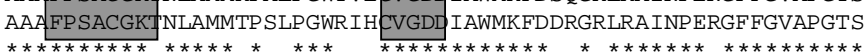

VKTNPNAIKTIQKNT IFTNVAETSDGGVYWEG IDEP LASGVT IT SWKNKEWS SEDGEPCA IKTNP NAIKTIF KNT IFTNVAE TSD GGVYWEG IDEP LP P GVTLT SWKNKDWTPDNGEP CA LKTNP HAMATIAKNTVFTNVGE TSDGGVWWEGLDPPAAG-VSLTDWHGKSWKAGD SGPCA SRTNPNAMATIARNT IFTNVGLRSDGGVYWDGLDEP TEPGVTYT SWLGKPWKHGDPEPCA

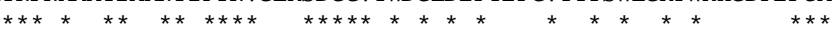

HPNSRFCTPASQCP I IDAAWE SPEGVPIEGI I FGGRRP AGVP LVYEALSWQHGVFVGAAM HPNSRF CTPASQCP IMDP AWESPEGVPIEGI I FGGRRP A GVP LVYEAFNWQHGVF IGAAM HPNSRFCTPAAQCP I I DP QWESDEGVPIDAI I FGGRRP E GVP LVYESFNWRHGVFVGASM HPNSRF CAP ADQCP IMDP RWDDPEGVP IDAI I FGGRRP RGVP LVVEAF GWRHGVF MGSAM

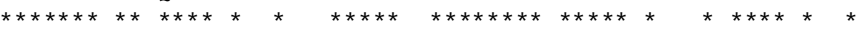

PEPCK-C-human PEPCK-C-chicken PEPCK-trout PEPCK-M-chicken

PEPCK-C-human PEPCK-C-chicken PEPCK-trout PEPCK-M-chicken

PEPCK-C-human PEPCK-C-chicken PEPCK-trout PEPCK-M-chicken
RSEATAAAEHKGKI IMHDP FAMRP F F GYNFGKYLAHWLSMAQHP-AAKLPKIFHVNWFRK RSEATAAAEHKGKI IMHDP FAMRP F F GYNFGKYLAHWLSMAHRP-AAKLPRIFHVNWFRK RSEATAAAEYKGKVIMHDPFAMRPFFGYNFGDYLAHWLSMETRKGATHLPK IFHVNWFRK RSEATAAAEHKGGRLMHDPFAMSP FFGYNAGRYLEHWLSTGLRS-NARLPRLFHVNWF LR

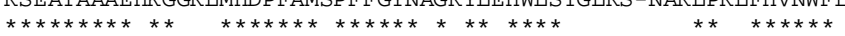

D-KEGKFLWPGF GENSRVLEWMFNR IDG---KAS TKLTP IGY IP KEDALNLKGLG-H INM D-SQGKFLWPGYGENSRVLEWMFNRIQG---KASAKSTAIGY IPADTALNLKGLE-D INL DP TSGSFLWPGFGDNARVLEWIFKRCSREREDEAAKKSMVGWVP LEGA INLQGLGSKVDM D-NEGRFVWPGF GHNARVLAWIFGRIQG---RDTARPTP IGWVP KEGD LDLGGLP-GVDY

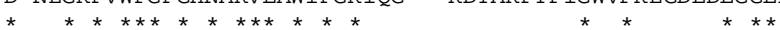

MELFS I SKEFWEKEVEDIEKYLEDQVNADLPCEIEREI LALKQRISQM TELFNI SKEFWEKEVEEIKQYFEGQVNADLPYEIERELLALEMRIKQL GALFDLPKAFWEKETQELRAYFTQQVGADLPQQVEGELKALEDRIRNSQLFPMEKGFWEEECRQLREYYGENFGADLPRDVMAELEGLEERVRKM

$\star \star \star \star * \star * \star * * \star * \star * *$

homologous to known PEPCK protein (BLAST algorithm, $P<10^{-179}$ ).

To obtain the full-length cDNA sequence data for PEPCK from trout, we used an established strategy (RACE-PCR). We obtained the full-length sequence information from two substantially overlapping $5^{\prime}$ and $3^{\prime}$ fragments of a given cDNA (Fig. 1). The initiator codon, arbitrarily fixed on the second ATG (the first ATG is followed by a stop codon five residues downstream), was followed by an open reading frame of 635 amino acid residues for rainbow trout PEPCK (Fig. 1). The reading frame of 420 codons unambiguously highly primers (Table 1) based on conserved sequences of known major (approximately $1300 \mathrm{bp}$ ) was obtained (data not shown). The fragments were purified, cloned and sequenced. The cDNA sequence of $1262 \mathrm{bp}$ was very similar to those of genes from other PEPCK genes (BLAST algorithm, $P=3 \times 10^{-30}$ to $\left.10^{-10}\right)$. The corresponding amino acid sequences were 
Fig. 3. Tissue specificity of rainbow trout phosphoenolpyruvate carboxykinase (PEPCK) gene expression in fish fed with carbohydrates and in fasted fish demonstrated by RT-PCR analysis. M, molecular mass marker (Promega, USA). Control, negative control (RT-PCR without cDNA).

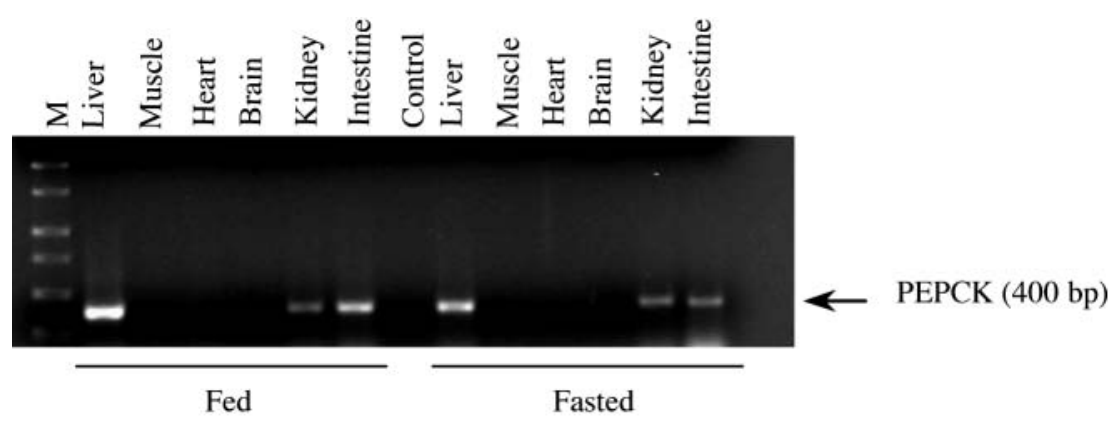

amino acid and nucleotide sequences, when compared with sequence databases using the BLAST algorithm, correspond unequivocally to PEPCK sequences except in the $5^{\prime}$ and $3^{\prime}$ untranslated regions (146 bp and $568 \mathrm{bp}$, respectively). The deduced teleost amino acid sequence for PEPCK was aligned with the human liver cytosolic PEPCK sequence and with cytosolic and mitochondrial PEPCK sequences from chicken (Fig. 2). The trout PEPCK sequence is very similar to PEPCK sequences from these higher vertebrates (67\% similarity with the human PEPCK). However, the data on trout PEPCK alone

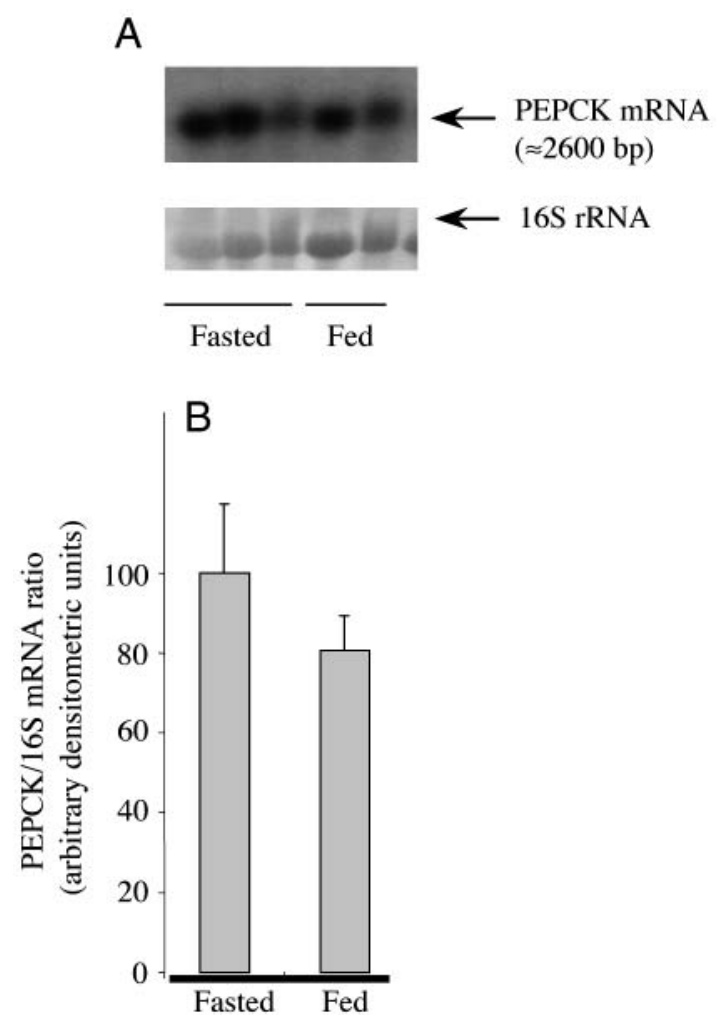

Fig. 4. (A) Rainbow trout phosphoenolpyruvate carboxykinase (PEPCK) gene expression in the liver of fasted and fed fish (6 h after feeding) (representative northern blot). The 16S rRNA served as an internal control for loading. The size of the PEPCK cDNA in trout is almost $2600 \mathrm{bp}$. (B) An analysis, by densitometry, of PEPCK mRNAs levels for five fish from each of the two treatment groups normalised to $16 \mathrm{~S}$ rRNA values (Visiomic II software). Values are means + S.D. The differences between groups were not significant (Student's $t$-test). do not allow us to discriminate between the cytosolic and the mitochondrial forms.

The tissue specificity of trout PEPCK gene expression was analysed by RT-PCR, which is more sensitive than northern blot analysis, and revealed that PEPCK gene expression is highly specific to liver, kidney and intestine irrespective of the nutritional status of the fish (Fig. 3). The derived PEPCK cDNA size of $2637 \mathrm{bp}$ for rainbow trout PEPCK cloned in this study (Fig. 1), without the poly(A) ${ }^{+}$tail, is in agreement with that deduced from northern blot analysis of mRNA (a unique fragment of almost $2600 \mathrm{bp}$ ) from this species (Fig. 4). We also observed that there was the same level of PEPCK mRNAs in fed and unfed fish (Fig. 4). Moreover, our results showed high and constant levels of PEPCK gene expression in the liver of fish fed with $(20 \%)$ or without carbohydrates for 10 weeks at $6 \mathrm{~h}$ and $24 \mathrm{~h}$ after feeding (Fig. 5A), indicating that hepatic PEPCK gene expression was not dependent on the presence of dietary carbohydrates (Fig. 5B).

\section{Discussion}

PEPCK belongs to a family of enzymes, including L-type pyruvate kinase (E.C. 2.7.1.40), glucokinase (E.C. 2.7.1.1) and fatty acid synthetase (E.C. 2.3.1.85), known to be regulated by dietary carbohydrates in mammals (Pilkis and Granner, 1992; Girard et al., 1997). The high similarity (up to $67 \%$ ) between cDNA nucleotide sequences of PEPCK in rainbow trout and PEPCK sequences from other vertebrates (Hanson and Reshef, 1997) confirms that this sequence corresponds to a functional enzyme; thus, trout PEPCK appears to possess the PEPCKspecific domain to bind oxaloacetate as well as kinase- 1 and kinase-2 motifs (see Fig. 2) to bind the GTP triphosphate chain and $\mathrm{Mg}^{2+}$, respectively, specific for all the known PEPCKs (Matte et al., 1997). Finally, the main gluconeogenic tissues (liver and kidney) and the intestine express the trout PEPCK gene, as in mammals (Hanson and Reshef, 1997). These data also confirm previous data on PEPCK activities that are highly specific to liver and kidney in fish (Suarez and Mommsen, 1987).

We observed that there was no significant effect of dietary carbohydrates on PEPCK gene expression, with a high level of PEPCK gene expression irrespective of the nutritional status of the fish (fed or unfed; fed with or without carbohydrates). These results contrast with those generally observed in mammals (Hanson and Reshef, 1997). However, PEPCK is 
A
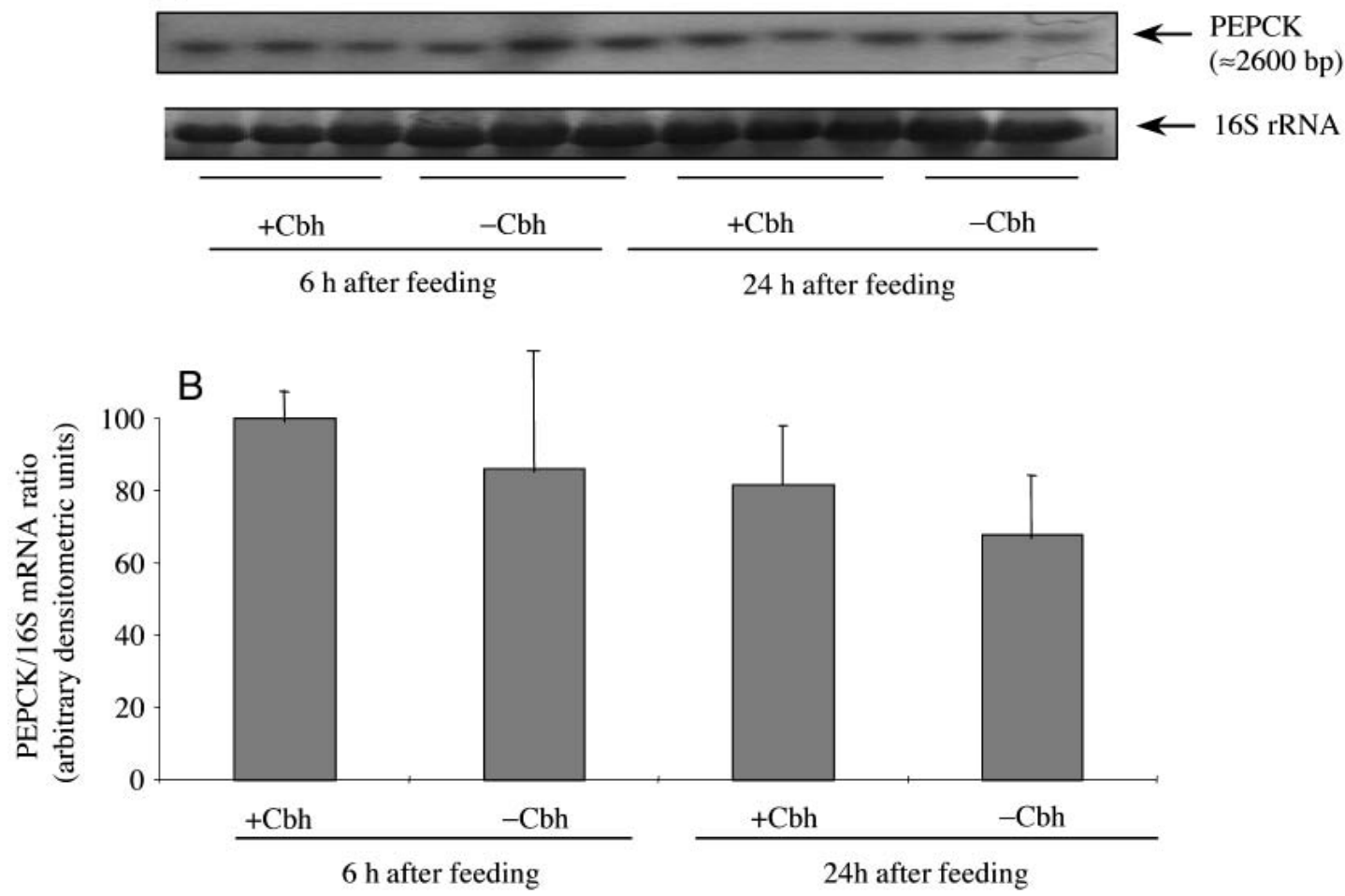

Fig. 5. Rainbow trout phosphoenolpyruvate carboxykinase (PEPCK) gene expression in the liver of fish fed with (+Cbh) or without (-Cbh) carbohydrates $6 \mathrm{~h}$ and $24 \mathrm{~h}$ after feeding. (A) A representative northern blot. Each band is from a different fish. The 16S rRNA served as an internal control for loading. (B) Analysis by densitometry of PEPCK mRNA levels for five fish from each of the two treatment groups normalised to $16 \mathrm{~S}$ rRNA values (Visiomic II software). Values are means + S.D. The differences between groups were not significant (one-way ANOVA).

known to occur as two isoforms (the mitochondrial and the cytosolic forms) with a different distribution and regulation pattern in different species (Hanson and Reshelf, 1997). In animals in which both the mitochondrial and the cytosolic forms occur, such as in chicken (Weldon et al., 1990), only the cytosolic form is acutely regulated by diet and hormones, whereas the gene for mitochondrial PEPCK is largely constitutive in its pattern of expression (for a review, see Hanson and Reshelf, 1997). On the basis of our data showing that PEPCK gene expression is not controlled by nutritional status, we suggest that the PEPCK gene cloned in the present study codes for a mitochondrial enzyme. This is supported by previous data (Mommsen and Suarez, 1984) showing that hepatic PEPCK activity in rainbow trout is mainly mitochondrial. A putative peptide signal from the first 35 amino acid residues of the trout PEPCK sequence (Fig. 2) is enriched in positively charged and hydroxylated residues (such as serine), specific to a mitochondrial-type signal (Neupert, 1997). If, indeed, rainbow trout possess only the mitochondrial PEPCK, this situation mimics that in birds, a unique case in animals studied so far. In birds, this is thought to be due to a specific metabolic adaptation: avian flight muscle goes through periods of prolonged exercise during which the cycling of lactate to glucose is essential, and the avian liver supports this critical metabolic function by expressing high levels of mitochondrial PEPCK (Weldon et al., 1990; Hanson and Reshelf, 1997). It has further been proposed that, in rainbow trout, the liver participates mainly in Cori cycle activity (through gluconeogenesis from lactate) catalysed by the constitutive mitochondrial PEPCK enzyme (Mommsen and Suarez, 1984), a situation again similar to that in birds. Consequently, the cloning of a putative hepatic mitochondrial PEPCK cDNA highly expressed in trout is, perhaps, to be expected.

The existence of a major form of PEPCK not inhibited by dietary carbohydrates can be compared with a similar absence of effects of dietary carbohydrate (i) on glucose-6-phosphatase activity and gene expression in the same species (Panserat et al., 2000b) and (ii) on fructose bisphosphatase (E.C. 3.1.3.11) activity in Atlantic salmon (Salmo salar) and perch (Perca fluviatilis) (Tranulis et al., 1996; Borrebaek and Christophersen, 2000). Taken together, these data suggest that the gluconeogenic pathway is not tightly controlled by dietary carbohydrates in rainbow trout. This, in itself, is not surprising since the natural diet of rainbow trout is almost devoid of carbohydrates, and glucose is produced from non-carbohydrate precursors (Suarez and Mommsen, 1987). We therefore suggest that the poor utilisation by rainbow trout of excess dietary carbohydrates is exacerbated by 'persistent' endogenous glucose production. Interestingly, our data for 
trout are similar to data for the carnivorous barn owl (Tyto alba), in which high rates of gluconeogenesis (including PEPCK activity) contribute to persistent hyperglycaemia after glucose challenge (Myers and Klasing, 1999).

In conclusion, following dietary carbohydrate intake, the observations of (i) persistent gluconeogenic expression of PEPCK, as seen here, and of glucose-6-phosphatase (Panserat et al., 2000b) and (ii) induction of glycolytic enzymes such as glucokinase (E.C. 2.7.1.1), pyruvate kinase (E.C. 2.7.1.40) and the bifunctional enzyme 6-phosphofructo-2-kinase/fructose2,6-biphosphatase (E.C. 2.7.1.105/E.C. 3.1.3.46) (Panserat et al., 2000a; S. Panserat, E. Plagnes-Juan and S. Kaushik, unpublished data) suggest that the adaptation of hepatic glucose metabolism to dietary glucose is not totally effective in trout, in contrast to mammals (Pilkis and Granner, 1992). It is worth investigating the effects of other nutritional or endocrine factors both in vivo and in vitro.

We acknowledge F. Terrier, F. Sandres and Y. Hontang for their help in the growth studies at the experimental facilities. The Genbank accession number for rainbow trout PEPCK sequence is AF246149.

\section{References}

Altschul, S., Gish, W., Miller, W., Myers, E. and Lipman, S. (1990). Basic local alignment search tool. J. Mol. Biol. 215, 403-410.

Borrebaek, B. and Christophersen, B. (2000). Hepatic glucose phosphorylation activities in perch (Perca fluviatilis) after dietary treatments. Comp. Biochem. Physiol. 125B, 387-393.

Chomczinski, P. and Sacchi, M. (1987). Single step method of RNA isolation by acid guanidium thiocyanate phenol chloroform extraction. Analyt. Biochem. 162, 156-159.

Cournarie, F., Azzout-Marniche, D., Foretz, M., Guichard, C., Ferre, P. and Foufelle, F. (1999). The inhibitory effect of glucose on phosphoenolpyruvate carboxykinase gene expression in cultured hepatocytes is transcriptional and requires glucose metabolism. FEBS Lett. 460, 527-532.

Cowey, C. B. and Walton, M. J. (1989). Intermediary metabolism. In Fish Nutrition (ed. E. Halver), pp. 259-329. New York: Academic Press.

Girard, J., Ferré, P. and Foufelle, F. (1997). Mechanisms by which carbohydrates regulate expression of genes for glycolytic and lipogeneic enzymes. Annu. Rev. Nutr. 17, 325-352.

Hanson, R. W. and Reshef, L. (1997). Regulation of phosphoenolpyruvate carboxykinase (GTP) gene expression. Annu. Rev. Biochem. 66, 581-611.

Higgins, D. and Sharp, P. (1989). Fast and sensitive multiple sequence alignments on a microcomputer. CABIOS 5, 151-153.

Matte, A., Tari, L. W., Goldie, H. and Delbaere, T. J. (1997).
Structure and mechanism of phosphoenolpyruvate carboxykinase. J. Biol. Chem. 272, 8105-8108.

Mommsen T. P. and Suarez, R. K. (1984). Control of gluconeogenesis in rainbow trout hepatocytes: role of pyruvate branchpoint and phosphoenolpyruvate-pyruvate cycle. Mol. Physiol. 6, 9-18.

Myers, M. R. and Klasing, K. C. (1999). Low glucokinase activity and high rates of gluconeogenesis contribute to hyperglycemia in barn owls (Tyto alba) after a glucose challenge. J. Nutr. 129, 1896-1904.

Neupert, W. (1997). Protein import into mitochondria. Annu. Rev. Biochem. 66, 863-917.

Nielsen, H., Engelbrecht, J., Brunak, S. and von Heijne, G. (1997). Identification of prokaryotic and eukaryotic signal peptides and prediction of their cleavage sites. Prot. Eng. 10, 1-6.

Panserat, S., Médale, F., Blin, C., Brèque, J., Vachot, C., PlagnesJuan, E., Krishnamoorthy, R. and Kaushik, S. (2000a). Hepatic glucokinase is induced by dietary carbohydrates in rainbow trout, common carp and gilthead seabream. Am. J. Physiol. 278, R1164-R1170.

Panserat, S., Médale, F., Brèque, J., Plagnes-Juan, E. and Kaushik, S. (2000b). Lack of significant long-term effect of dietary carbohydrates on glucose-6-phosphatase expression in liver of rainbow trout (Oncorhynchus mykiss). J. Nutr. Biochem. 11, 22-29.

Parrizas, M., Planas, J., Plisetskaya, E. M. and Gutierrez, J. (1994). Insulin binding and receptor tyrosine kinase activity in skeletal muscle of carnivorous and omnivorous fish. Am. J. Physiol. 266, R1944-R1950.

Pilkis, S. J. and Granner, D. K. (1992). Molecular physiology of the regulation of hepatic gluconeogenesis and glycolysis. Annu. Rev. Physiol. 54, 885-909.

Scott, D. K., O'Doherty, R. M., Stafford, J. M., Newgard, C. B. and Granner, D. K. (1998). The repression of hormone-activated PEPCK gene expression by glucose is insulin-independent but requires glucose metabolism. J. Biol. Chem. 273, 24145-24151.

Suarez, R. K. and Mommsen, T. P. (1987). Gluconeogenesis in teleost fishes. Can. J. Zool. 65, 1869-1882.

Tranulis, M. A., Dregni, O., Cristophersen, B., Krogdahl, A. and Borrebaek, B. (1996). A glucokinase-like enzyme in the liver of Atlantic salmon (Salmon salar). Comp. Biochem. Physiol. 114B, 35-39.

Valera, A., Pujol, A., Pelegrin, M. and Bosh, F. (1994). Transgenic mice overexpressing phosphoenolpyruvate carboxykinase develop non-insulin-dependent diabetes mellitus. Proc. Natl. Acad. Sci. USA 91, 9151-9154.

Weldon, S. L., Rando, A., Matathias, A. S., Hod, Y., Kalonick, P. A., Savon, S., Cook, J. S. and Hanson, R. W. (1990). Mitochondrial phosphoenolpyruvate carboxykinase from the chicken: comparison of the cDNA protein sequences with the cytosolic isozyme. J. Biol. Chem. 265, 7308-7317.

Wright, J. R., O'Hali, W., Yuang, H., Han, X. and Bonen, A. (1998). Glut-4 deficiency and severe peripheral resistance to insulin in the teleost fish tilapia. Gen. Comp. Endocr. 111, 20-27. 